\title{
Phosphorus P-32
}

National Cancer Institute

\section{Source}

National Cancer Institute. Phosphorus P-32. NCI Thesaurus. Code C1698.

A radioactive isotope of phosphorus with beta particle-emitting radiocytotoxic activity. Emitted by phosphorus P32, beta particles directly damage cellular DNA and, by ionizing intracellular water to produce several types of cytotoxic free radicals and superoxides, indirectly damage intracellular biological macromolecules, resulting in tumor cell death. 